\title{
Increased Risk of Atopic Dermatitis in Preschool Children with Kawasaki Disease: A Population-Based Study in Taiwan
}

\author{
Peng Yeong Woon, ${ }^{1}$ Wei Chiao Chang, ${ }^{2,3,4,5}$ Chi-Cheng Liang, ${ }^{6}$ Chun Hung Hsu, ${ }^{7}$ \\ Sukhontip Klahan, ${ }^{2}$ Ying-Hsien Huang, ${ }^{8}$ Wei-Pin Chang, ${ }^{7}$ and Ho-Chang Kuo ${ }^{8}$ \\ ${ }^{1}$ Department of Molecular Biology and Human Genetics, Tzu Chi University, Hualien 97004, Taiwan \\ ${ }^{2}$ Department of Clinical Pharmacy, Taipei Medical University, Taipei 110, Taiwan \\ ${ }^{3}$ Master Program for Clinical Pharmacogenomics and Pharmacoproteomics, \\ School of Pharmacy, Taipei Medical University, Taipei 110, Taiwan \\ ${ }^{4}$ Department of Pharmacy, Taipei Medical University-Wanfang Hospital, Taipei 110, Taiwan \\ ${ }^{5}$ Cancer Center, Kaohsiung Medical University Hospital, Kaohsiung Medical University, Kaohsiung 807, Taiwan \\ ${ }^{6}$ Division of Emergency and Trauma Surgery, Department of Surgery, Kaohsiung Chang Gung Memorial Hospital and Chang Gung \\ University, College of Medicine, Kaohsiung 83301, Taiwan \\ ${ }^{7}$ Department of Healthcare Management, Yuanpei University, Hsinchu 30015, Taiwan \\ ${ }^{8}$ Department of Pediatrics, Kaohsiung Chang Gung Memorial Hospital and Chang Gung University College of Medicine, \\ Kaohsiung 83301, Taiwan
}

Correspondence should be addressed to Wei-Pin Chang; wpchang@mail.ypu.edu.tw and

Ho-Chang Kuo; erickuo48@yahoo.com.tw

Received 4 March 2013; Revised 10 July 2013; Accepted 26 July 2013

Academic Editor: Xiu-Min Li

Copyright (C) 2013 Peng Yeong Woon et al. This is an open access article distributed under the Creative Commons Attribution License, which permits unrestricted use, distribution, and reproduction in any medium, provided the original work is properly cited.

\begin{abstract}
Kawasaki disease (KD) is an acute febrile systemic vasculitis and has been reported to be associated with allergic disease. The risk of atopic dermatitis (AD) in preschool children with KD has not been investigated. The study was to determine the longitudinal risk of the development of $\mathrm{AD}$ in preschool children with $\mathrm{KD}$. A nationwide 5-year population-based study was performed using data from the National Health Insurance Database in Taiwan between 1999 and 2003. The risk factors for AD were compared between the 2 study groups during the follow-up period using the Cox proportional hazards model. In addition, plasma interleukin (IL)-5 levels were analyzed in normal subjects and KD patients. Among the 1440 subjects included, 21.6\% developed AD during the 5-year follow-up period, of which $30.3 \%$ and $18.7 \%$ belonged to the study cohort and the comparison group, respectively. Children with KD were 1.25 times more likely to have AD than those in controls $(P=0.04)$. Levels of IL-5 and IgE were significantly higher in $\mathrm{KD}$ patients. Children with KD had a higher risk of developing AD during the 5-year follow-up period than the control group. Increased IL-5 and IgE levels may be key factors contributing to the risk of AD.
\end{abstract}

\section{Introduction}

Kawasaki disease $(\mathrm{KD})$ is an acute febrile systemic vasculitis that was first described by Kawasaki et al. [1]. In developed countries, it is the leading cause of acquired heart disease in children. However, the etiology of KD remains unknown [25]. The acute stage of $\mathrm{KD}$ is characterized by systemic inflammatory changes, including high-grade fever lasting $\geq 5$ days, bilateral conjunctivitis, oral mucosal changes, fissured lips, unilateral cervical lymphadenopathy $(>1.5 \mathrm{~cm})$, dysmorphic skin rash, and erythema of the palms and soles $[6,7]$. The most serious complication of $\mathrm{KD}$ is the involvement of coronary artery lesions (CALs), including myocardial infarction, coronary artery fistula formation [8], coronary artery dilatation, and coronary artery aneurysm (CAA) $[9,10]$. Children $<5$ years of age are the most susceptible population. According to recent epidemiologic studies, the incidence of $\mathrm{KD}$ is significantly higher in Asian populations. Japan has 
the highest annual incidence rates of $\mathrm{KD}$ in the world [11], followed by Korea [12] and Taiwan [13] (239, 113, and 69 per 100,000 children $<5$ years of age, resp.). The incidence of $\mathrm{KD}$ has increased globally in recent years [10, 14-17].

The prevalence of allergic diseases, including asthma, atopic dermatitis (AD), and allergic rhinitis, has increased worldwide in recent decades, primarily in developed countries [18-21]. This increase has been attributed to environmental factors, including improved hygiene, increased use of antimicrobial medications, consumption of sterile foods, and reduced family size, which have resulted in low rates of infection during childhood and less contact with microbes. Burns et al. [22] reported that the associations of $\mathrm{KD}$ with $\mathrm{AD}$, elevated serum IgE levels, eosinophilia, and increased circulating numbers of monocyte/macrophages expressing the low-affinity IgE receptor may be related to the effects of IL-4. Brosius et al. [23] showed that the incidence of AD among children with $\mathrm{KD}$ was 9 times greater than that among control subjects. Liew et al. [24] reported that KD may be a risk factor for subsequent allergic disease and postulated that KD occurs more frequently in children at risk of immune disequilibrium, with an initial abnormal inflammatory response and a subsequent increase in allergic manifestations. We previously reported that the $\mathrm{T}$ helper (Th) type 2 immune response was elevated in the acute stage of KD, and the involvement of eosinophils [25], interleukin-4 (IL-4), IL-5 [26], and eotaxin was shown. The changes in the levels of eosinophils correlated with changes in the levels of IL-5 but not eosinophil cationic protein (ECP), suggesting the involvement of a Th2 immune reaction in $\mathrm{KD}$. We also reported that genes involved in immune regulation (CTLA-4 and TGF-beta signaling pathway) were associated with $\mathrm{KD}$ with or without CAL formation [27, 28]. Importantly, both CTLA-4 and TGF-beta signaling pathways have been shown to be associated with the development of AD [29-31]. Indeed, an abnormal Th1/Th2 balance plays a key role in the development of $\mathrm{KD}$ [7, 25, 26, 32-35]. However, the association between preschool children with $\mathrm{KD}$ and $\mathrm{AD}$ has not been investigated to date.

The incidence of KD has increased $[17,36]$ in parallel with the increase in the incidence of allergic diseases worldwide [37]. In the present study, we examined the incidence of AD in patients with $\mathrm{KD}$ during a 5-year follow-up period using a nationwide population-based dataset obtained from Taiwan's National Health Insurance (NHI) health care database. We also analyzed the levels of IL-5, an important biomarker of allergic diseases, and detected high levels of IL- 5 expression in $\mathrm{KD}$ patients during the acute stage of the disease.

\section{Methods}

2.1. Database. The present study used data obtained from Taiwan's NHI database. Taiwan began the NHI program in 1995 as a mandatory plan that covered nearly $99 \%$ of the Taiwanese population of almost 23 million residents. The NHI program was organized by Taiwan's Bureau of National Health Insurance (BNHI). To encourage researchers to perform health studies on issues relevant to the NHI program, the BNHI created and released the Longitudinal Health
Insurance Database 2005 (LHID2005) to the public for research purposes.

Our study adopted the LHID2005, which included registration and medical claims for 1 million randomly sampled individuals from all the NHI enrollees ( $n=23$ million). The dataset included information on all medical claims of these 1 million beneficiaries between 1996 and 2010. The National Health Research Institute (NHRI) claims that there were no statistically significant differences in age, sex, or health care costs between the selected group and all enrollees. Because the LHID2005 dataset was established for research purposes, personal identifying information could not be obtained. As the NHRI had addressed the confidentiality assurance issue, the present study was exempted from full review by the Institutional Review Board.

2.2. Study Population. A total of 360 preschool KD children were considered eligible for the study. The inclusion criteria were age $\leq 6$ years and newly diagnosed KD (ICD-9-Code 446.1) between January 1999 and December 2003. Moreover, to ensure the diagnostic precision of the administrative dataset, we only included preschool children who had been diagnosed with $\mathrm{KD} \geq 2$ times during outpatient visits or $\geq 1$ time as inpatients. We excluded patients diagnosed with KD before 1999 ( $n=43$ ). Our study assigned each child a first diagnosis of KD as the index date between January 1999 and December 2003. Control groups were randomly selected from the remaining subjects of the LHID2005 and matched with a control-to-case ratio of $3: 1$ on the basis of age, sex, and index year.

Each child was tracked for 5 years from the index date to determine the incidence of AD (ICD-9-CM 691.8, 692.9). To improve diagnosis accuracy, the selection criteria required that all AD cases ICD-9 code was assigned by pediatrician or dermatologist. In addition, to investigate the relationship between $\mathrm{AD}$ and particular comorbidities, we analyzed certain covariables such as chronic obstructive pulmonary disease (COPD; ICD-9-CM 490.X-496.X), hyperlipidemia (ICD-9-CM 272.X), asthma (ICD-9-CM 493.X), allergic rhinitis (ICD-9-CM 477.X), and heart disease (ICD-9-CM 410.X-414.X) in our cohort study.

\subsection{Level of Urbanization and Geographic Region. Regarding} urbanization level, the Taiwanese National Health Research Institute performed cluster analysis to study urbanization levels, which resulted in the division of 359 towns or cities in Taiwan into 7 categories, with 1 representing "the most urbanized" and 7 representing "the least urbanized." These 7 categories were defined by incorporating various variables such as population density, proportion of elderly people and agriculture workers, number of physicians per 100,000 people, and different educational levels, which were based on the 2000 Taiwan census data. Only a small number of preschool children with KD were identified in levels 5, 6, and 7. Therefore, these 3 levels were combined into a single group. For the purposes of the study, we divided Taiwan into 4 geographic regions, northern, central, southern, and eastern Taiwan. Urbanization level and geographic region were incorporated 
into our model to adjust for possible differences in access to medical care.

2.4. Measurement of Interleukin-5 and IgE Levels. This study was approved by the Institutional Review Board of Chang Gung Memorial Hospital. Blood samples were collected after obtaining informed consent from the parents or guardians. Blood samples collected before intravenous immunoglobulin (IVIG) treatment (within 24 hours before IVIG treatment, acute stage) and after IVIG treatment (at least 3 weeks after IVIG treatment, subacute stage) were included in the analysis.

Plasma levels of IL-5 were assessed using the Upstate Beadlyte Human Cytokine Beadmates system (Upstate Group, Inc.) in 64 patients with KD patients and 14 controls, according to a modification of the method described previously [26]. In brief, $50 \mu \mathrm{L}$ of plasma was mixed with multiplexed antibody-conjugated beads and subjected to multichannel detection of the bead array. Acquired fluorescence data were assessed using the MasterPlex TM QT software (Ver. 1.2; MiraiBio, Inc.). Cytokine concentrations were calibrated by interpolation of a series of well-known standard samples following the manufacturer's recommendations. The assay sensitivity of these cytokines was $0.2 \mathrm{pg} / \mathrm{mL}$. To avoid interassay bias in the immunoassays, the cytokines in paired samples before and after IVIG therapy were measured at the same time. IgE levels were quantified by a sensitive, IgE detection system with the automatic Pharmacia UnicAP device (Pharmacia \& Upjohn Diagnostics AB, Uppsala, Sweden) using a fluorescence enzyme immunoassay system.

2.5. Statistical Analysis. The SPSS (Statistical Package for Social Science) statistical software version 18.0 (SPSS, Inc.) was used for data analysis. All data were expressed as frequency and percentage or mean and standard deviation. Chi-square test was performed to assess differences in geographic location and urbanization level of patients' residences between the study group and comparison group. Stratified Cox proportional hazard regression analysis (stratified by sex, age group, and year of index) was performed to investigate the risk of subsequent $\mathrm{AD}$ during the 5-year follow-up period in preschool children with and without $\mathrm{KD}$. All subjects were followed from the index date until the detection of $\mathrm{AD}$ or the end of the 5-year follow-up period. Hazard ratios (HRs) along with $95 \%$ confidence intervals (CIs) were calculated to determine the risk of colorectal cancer. Plasma levels of IL-5 were analyzed by the Mann-Whitney $U$ test. A 2 -sided $P$ value $<0.05$ was considered statistically significant.

\section{Results}

3.1. Correlation between $K D$ and $A D$. A total of 360 preschool children diagnosed with $\mathrm{KD}$ matched the inclusion criteria, and 1080 preschool children were included in the comparison cohort. Table 1 shows the demographic characteristics of the study subjects stratified by the presence of KD. Diseases such as COPD, hyperlipidemia, asthma, allergic rhinitis, and heart disease were associated with KD. Table 2 shows that among the 1440 patients comprising the study population, $\mathrm{AD}$ was detected in 311 subjects during the follow-up period. AD was

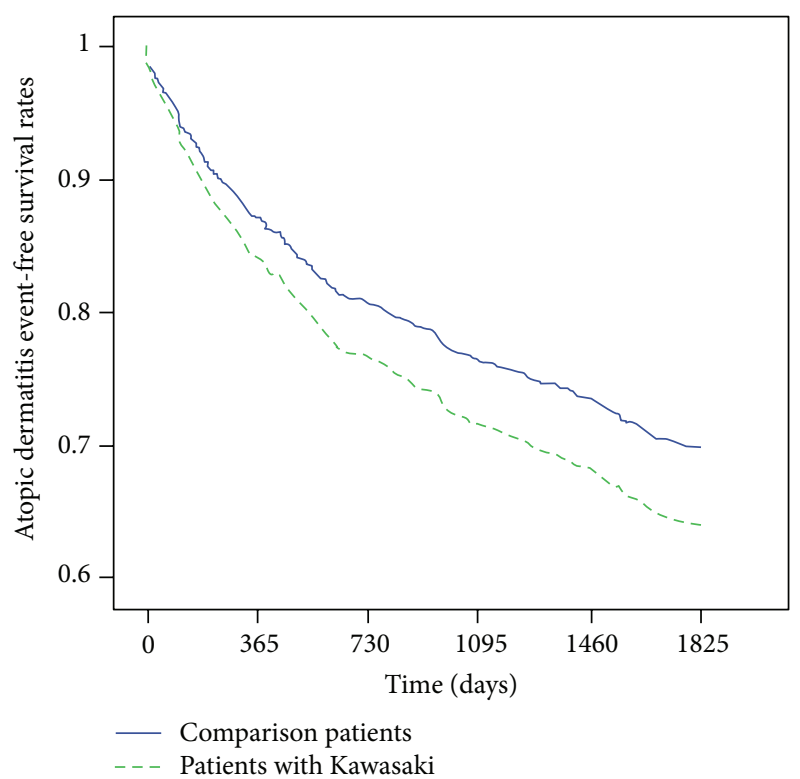

FIGURE 1: Event free survival rates of atopic dermatitis for children with Kawasaki disease and the comparison group between 1999 and 2003.

detected in 109 children with $\mathrm{KD}$, representing an incidence rate of 104.68 per 1000 person years, and in 202 subjects in the comparison cohort, representing an incidence rate of 76.04 per 1000 person years. Cox regression analysis also showed that the crude $\mathrm{HR}$ of $\mathrm{AD}$ was 1.43 times greater for children with $\mathrm{KD}$ than for the comparison cohort ( $95 \% \mathrm{CI}=1.11-1.85$; $P<0.01)$. The HR remained significant after adjusting for potential confounders (adjusted HR: $1.25,95 \% \mathrm{CI}=1.01-1.54$; $P<0.05)$. The results of survival analysis with the Cox regression model are shown in Figure 1.

\subsection{Expression Levels of IL-5 and IgE Were Significantly Higher} in KD Patients than Normal Subjects. Plasma levels of IL5 before IVIG treatment were significantly higher in KD patients $(n=64)$ than those in the controls $(n=14)(P=$ $0.001)$. Moreover, IL-5 levels were significantly lower in patients in the subacute stage of $\mathrm{KD}(n=64)$ than those in patients in the acute stage of $\mathrm{KD}(P<0.001)$ (Figure 2). We further tested the expression levels of IgE in KD patients and control subjects. As shown in Figure 3, high IgE expression level in KD patients was observed $(P=0.0003)$.

\section{Discussion}

This is the first population-based study to use a nationwide dataset to investigate the relation between $\mathrm{AD}$ and $\mathrm{KD}$ in children during a 5-year follow-up period. Our results indicated that children with $\mathrm{KD}$ have a higher risk of developing $\mathrm{AD}$ than healthy controls $(95 \% \mathrm{CI}=1.01-1.54 ; P<0.05)$.

$\mathrm{KD}$ is a systemic vasculitis of unknown etiology, and its immunopathogenesis remains unclear. Several lines of evidence have shown an imbalance between Th1 and Th2 immune reactions in $\mathrm{KD}$ patients. Th1 immune related 
TABLE 1: Demographic characteristics of the study groups, stratified by the presence or absence of Kawasaki disease between 1999 and 2003 $(n=1440)$.

\begin{tabular}{|c|c|c|c|c|c|}
\hline & Patients & ki disease $(n=360)$ & Patients w & aki disease $(n=1080)$ & $P$ value \\
\hline & $n$ & Percentage & $n$ & Percentage & 1 varue \\
\hline Gender & & & & & 1 \\
\hline Male & 194 & 53.9 & 582 & 53.9 & \\
\hline Female & 166 & 46.1 & 498 & 46.1 & \\
\hline Age (years) & & & & & 1 \\
\hline 0 & 59 & 16.4 & 177 & 16.4 & \\
\hline 1 & 121 & 33.6 & 361 & 33.4 & \\
\hline 2 & 67 & 18.6 & 203 & 18.8 & \\
\hline 3 & 38 & 10.6 & 113 & 10.5 & \\
\hline 4 & 37 & 10.3 & 112 & 10.4 & \\
\hline 5 & 20 & 5.6 & 62 & 5.7 & \\
\hline 6 & 18 & 5.0 & 52 & 4.8 & \\
\hline Urbanization level & & & & & 0.03 \\
\hline 1 (most urbanized) & 111 & 30.8 & 257 & 23.8 & \\
\hline 2 & 128 & 35.6 & 384 & 35.6 & \\
\hline 3 & 51 & 14.2 & 197 & 18.2 & \\
\hline 4 (least urbanized) & 70 & 19.4 & 242 & 22.4 & \\
\hline Geographic region & & & & & 0.41 \\
\hline Northern & 192 & 53.3 & 526 & 48.7 & \\
\hline Central & 96 & 26.7 & 304 & 28.1 & \\
\hline Southern & 64 & 17.8 & 216 & 20.0 & \\
\hline Eastern & 8 & 2.2 & 34 & 3.1 & \\
\hline COPD & & & & & $<0.001$ \\
\hline Yes & 212 & 58.9 & 503 & 46.6 & \\
\hline No & 148 & 41.1 & 577 & 53.4 & \\
\hline Hyperlipidemia & & & & & 0.01 \\
\hline Yes & 6 & 1.7 & 4 & 0.4 & \\
\hline No & 354 & 98.3 & 1076 & 99.6 & \\
\hline Asthma & & & & & $<0.001$ \\
\hline Yes & 177 & 49.2 & 405 & 37.5 & \\
\hline No & 183 & 50.8 & 675 & 62.5 & \\
\hline Allergic rhinitis & & & & & $<0.001$ \\
\hline Yes & 243 & 67.5 & 612 & 56.7 & \\
\hline No & 117 & 32.5 & 468 & 43.3 & \\
\hline Heart disease & & & & & $<0.001$ \\
\hline Yes & 53 & 14.7 & 29 & 2.7 & \\
\hline No & 307 & 85.3 & 1051 & 97.3 & \\
\hline
\end{tabular}

responses (IFN-gamma, tumor necrosis factor-alpha, IL$1 \beta$, and IL-10) $[38,39]$ and Th2 immune related responses (eosinophils, IL-4 [22], IL-5 [25, 26], eotaxin, and total IgE) have been reported to be associated with the susceptibility to $\mathrm{KD}$ and disease outcomes. Furthermore, serum IgE levels were shown to be increased in KD patients during the acute stage of the disease [34]. Therefore, an association between $\mathrm{KD}$ and allergic diseases has been suggested.

IL-5 and IL-5 receptor alpha polymorphisms were reported to be associated with AD [40]. IL-5 and Th2 cells promote AD development mediated by CCL8 [41]. Elevated numbers of blood and tissue eosinophils are present in allergic diseases and KD [25], suggesting that eosinophils play an important pathogenic role in these diseases. The regulation of eosinophil maturation, recruitment, and survival is controlled by a group of factors, including IL-5. Therefore, IL-5 has been proposed as a potential molecular target in the treatment of allergic diseases [42]. In the present study, IL-5 was found to be expressed at higher levels in $\mathrm{KD}$ patients in the early stage of KD. IVIG treatment resulted in 
TABLE 2: Hazard ratios (HRs) of atopic dermatitis among patients with Kawasaki disease during a 5-year follow-up period from the first ambulatory visit or inpatient care between 1999 and 2003.

\begin{tabular}{|c|c|c|c|c|c|c|}
\hline \multirow{2}{*}{ Development of atopic dermatitis } & \multicolumn{2}{|c|}{ Total } & \multicolumn{2}{|c|}{ Patients with Kawasaki disease } & \multicolumn{2}{|c|}{ Patients without Kawasaki disease } \\
\hline & No. & (Percentage) & No. & (Percentage) & No. & (Percentage) \\
\hline \multicolumn{7}{|l|}{ Five-year follow-up period } \\
\hline Yes & 311 & 21.6 & 109 & 30.3 & 202 & 18.7 \\
\hline No & 1129 & 78.4 & 251 & 69.7 & 878 & 81.3 \\
\hline Crude HR (95\% CI) & & & \multicolumn{2}{|c|}{$1.43(1.11-1.85)^{* *}$} & 1 & \\
\hline Adjusted HR (95\% CI) & & & \multicolumn{2}{|c|}{$1.25(1.01-1.54)^{*}$} & 1 & \\
\hline
\end{tabular}

Total sample number $=1440$.

Crude and adjusted HRs were calculated using Cox proportional hazard regression.

Adjustments were made for patients' urbanization level, geographic region, COPD, hyperlipidemia, asthma, allergic rhinitis, and heart disease.

* Indicates that $P<0.05$; $^{* *}$ indicates that $P<0.01$.

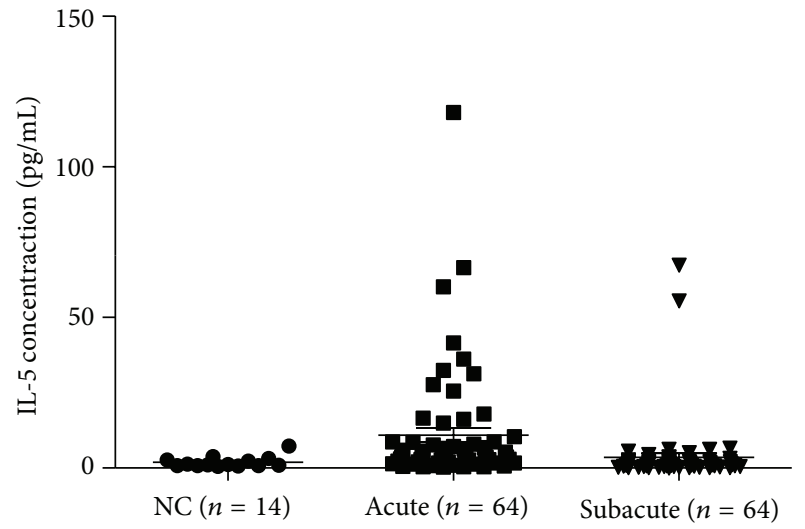

FIGURE 2: Plasma levels of interleukin-5 (IL-5) were detected in normal controls $(n=14)$, patients with acute $\mathrm{KD}(n=64$, before IVIG treatment), and subacute $\mathrm{KD}(n=64$, at least 3 weeks after IVIG treatment). Patients with acute KD showed significantly higher levels of IL-5 than normal controls.

a significant decrease in IL-5 levels. Recent studies indicated that keratinocyte TSLP and IL-33 are generated by the damaged $\mathrm{AD}$ barrier and trigger Th2 responses $[43,44]$. Thus, this same mechanism may account for increased Th2 responses during the early phases of $\mathrm{KD}$.

The molecular mechanisms common to $\mathrm{AD}$ and $\mathrm{KD}$ are still unclear. Previous genetic polymorphism studies in Japanese and Taiwanese populations indicated that genetic variants of the ORAI1 gene, which encodes a membrane calcium channel subunit, contribute to the susceptibility to atopic dermatitis [45]. Although an association between genetic polymorphisms of ORAIl and the risk of KD was not observed in a Taiwanese population study [46], inositol 1,4,5trisphosphate 3-kinase C (ITPKC) gene, a gene upstream of ORAI1, was shown to be an important biomarker for the susceptibility to KD [10]. The polymorphism of ITPKC (rs28493229) altered the transcriptional level of the mature mRNA by interfering with the RNA splicing efficiency [47]. ITPKC protein phosphorylates inositol 1,4,5-trisphosphate $\left(\mathrm{IP}_{3}\right)$, which plays a role in ORAIl-mediated immune responses [39]. Thus, ITPKC-mediated calcium signaling

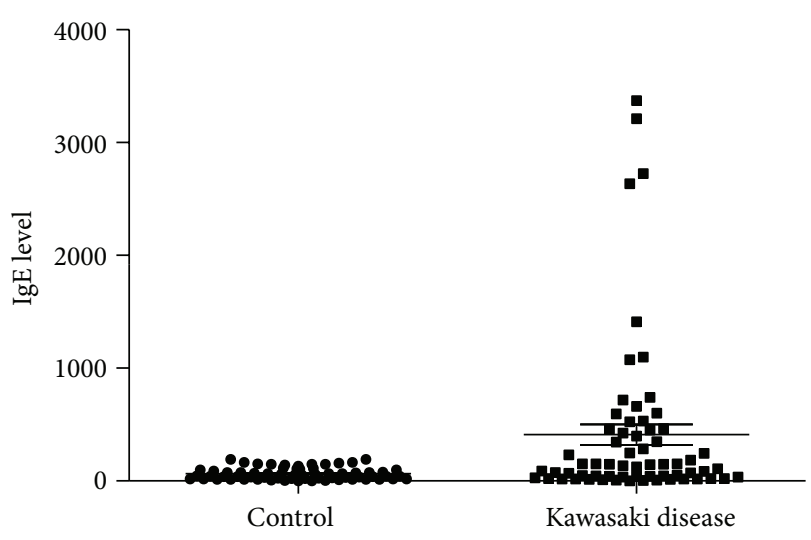

FIgURE 3: Expression levels of IgE were detected in normal controls $(n=64)$ and patients with $\mathrm{KD}(n=64)$. Patients with KD showed significantly higher levels of IgE than normal controls.

may be a common molecular mechanism underlying $\mathrm{KD}$ and $\mathrm{AD}$.

The incidence of $\mathrm{AD}$ among children with $\mathrm{KD}$ has been reported to be greater than that of control subjects and was associated with higher serum IgE concentrations [19]. Indeed, we also observed high-expression level of IgE in KD patients in this study. Another study that included Japanese children reported a higher incidence of $\mathrm{AD}$ in children with $\mathrm{KD}$ than in healthy controls, even among children with no family history of allergies [33]. In addition, $\mathrm{KD}$ may be a risk factor for subsequent allergic disease and therefore may be associated with allergic manifestations [20]. Consistent with the results of previous studies, our results indicated that children with $\mathrm{KD}$ were more likely to develop $\mathrm{AD}$ than healthy children.

Our study had few limitations. First, certain factors such as the incidence of household pets, cigarette smoking in the family, or the family history of allergy, which could affect the susceptibility to the development of $\mathrm{AD}$ in children, were not included in the analysis. Second, household income and economic status are other indexes besides urbanization or geographic region that could be important for the evaluation of medical access or medical utilization. Third, the expression 
levels of IL-5 and IgE in AD patients should be evaluated to further confirm the association between $\mathrm{AD}$ and $\mathrm{KD}$ in the Taiwanese pediatric population. Despite these limitations, the use of a nationwide dataset, which provided a large sample size, and adjustment for certain covariates related to $\mathrm{KD}$ enabled the detection of a significant relation between $\mathrm{KD}$ in preschool children and the risk of developing AD during the 5-year follow-up period.

\section{Conclusion}

In the present study, we showed that $\mathrm{KD}$ is associated with the risk of $\mathrm{AD}$ in preschool children, although the underlying mechanisms remain unclear. Increased levels of IL-5 and IgE may be key factors contributing to the risk of AD. Further studies are required to verify our results and to include related physiological variables.

\section{Conflict of Interests}

The authors declare that there is no conflict of interests.

\section{Authors' Contribution}

Peng Yeong Woon and Wei Chiao Chang contributed equally to this paper.

\section{Acknowledgments}

This study is based in part on data from the National Health Insurance Research Database provided by the Bureau of National Health Insurance, Department of Health, and managed by the National Health Research Institutes, Taiwan. The results and their interpretation do not represent those of the Bureau of National Health Insurance, Department of Health, or National Health Research Institutes. This study was partly supported by grants from the National Science Council, Taiwan (NSC 100-2314-B-182-061-MY3; NSC101-2628-B038001-MY2; NSC101-2320-B038-029-MY3), a grant from Chang Gung Memorial Hospital (CMRPG8A0481), and a grant from NTUT-TMU-102-16.

\section{References}

[1] T. Kawasaki, F. Kosaki, S. Okawa, I. Shigematsu, and H. Yanagawa, "A new infantile acute febrile mucocutaneous lymph node syndrome (MLNS) prevailing in Japan," Pediatrics, vol. 54, no. 3, pp. 271-276, 1974.

[2] C.-L. Wang, Y.-T. Wu, C.-A. Liu, H.-C. Kuo, and K. D. Yang, "Kawasaki disease: infection, immunity and genetics," Pediatric Infectious Disease Journal, vol. 24, no. 11, pp. 998-1004, 2005.

[3] J. C. Burns and M. P. Glodé, "Kawasaki syndrome," The Lancet, vol. 364, no. 9433, pp. 533-544, 2004.

[4] H.-C. Kuo, C.-D. Liang, C.-L. Wang, H.-R. Yu, K.-P. Hwang, and K. D. Yang, "Serum albumin level predicts initial intravenous immunoglobulin treatment failure in Kawasaki disease," Acta Paediatrica, International Journal of Paediatrics, vol. 99, no. 10, pp. 1578-1583, 2010.
[5] H.-C. Kuo, K. D. Yang, W.-C. Chang, L.-P. Ger, and K.-S. Hsieh, "Kawasaki disease: an update on diagnosis and treatment," Pediatrics and Neonatology, vol. 53, no. 1, pp. 4-11, 2012.

[6] C.-D. Liang, H.-C. Kuo, K. D. Yang, C.-L. Wang, and S.-F. Ko, "Coronary artery fistula associated with Kawasaki disease," American Heart Journal, vol. 157, no. 3, pp. 584-588, 2009.

[7] H.-C. Kuo, C.-L. Wang, L. Wang, H.-R. Yu, and K. D. Yang, "Patient characteristics and intravenous immunoglobulin product may affect eosinophils in Kawasaki disease," Pediatric Allergy and Immunology, vol. 19, no. 2, pp. 184-185, 2008.

[8] R. G. Townley, I. B. Barlan, C. Patino et al., "The effect of BCG vaccine at birth on the development of atopy or allergic disease in young children," Annals of Allergy, Asthma and Immunology, vol. 92, no. 3, pp. 350-355, 2004.

[9] J. W. Newburger, M. Takahashi, M. A. Gerber et al., "Diagnosis, treatment, and long-term management of Kawasaki disease: a statement for health professionals from the Committee on Rheumatic Fever, Endocarditis and Kawasaki Disease, Council on Cardiovascular Disease in the Young, American Heart Association," Circulation, vol. 110, no. 17, pp. 2747-2771, 2004.

[10] H.-C. Kuo, K. D. Yang, S.-H. H. Juo et al., "Itpkc single nucleotide polymorphism associated with the kawasaki disease in a taiwanese population," PLoS ONE, vol. 6, no. 4, Article ID e17370, 2011.

[11] Y. Nakamura, M. Yashiro, R. Uehara et al., "Epidemiologic features of Kawasaki disease in Japan: results of the 2009-2010 nationwide survey," Journal of Epidemiology, vol. 22, no. 3, pp. 216-221, 2012.

[12] Y. W. Park, J. W. Han, Y. M. Hong et al., "Epidemiological features of Kawasaki disease in Korea, 2006-2008," Pediatrics International, vol. 53, no. 1, pp. 36-39, 2011.

[13] W.-C. Huang, L.-M. Huang, I.-S. Chang et al., "Epidemiologic features of Kawasaki disease in Taiwan, 2003-2006," Pediatrics, vol. 123, no. 3, pp. e401-e405, 2009.

[14] Z.-D. Du, D. Zhao, J. Du et al., "Epidemiologic study on Kawasaki disease in Beijing from 2000 through 2004," Pediatric Infectious Disease Journal, vol. 26, no. 5, pp. 449-451, 2007.

[15] M. Bar-Meir, Z. Haklai, and M. Dor, "Kawasaki disease in Israel," Pediatric Infectious Disease Journal, vol. 30, no. 7, pp. 589-592, 2011.

[16] S. Singh, R. Aulakh, A. K. Bhalla et al., "Is Kawasaki disease incidence rising in Chandigarh, North India?" Archives of Disease in Childhood, vol. 96, no. 2, pp. 137-140, 2011.

[17] Y. Nakamura, M. Yashiro, R. Uehara et al., "Epidemiologic features of Kawasaki disease in Japan: results of the 2007-2008 nationwide survey," Journal of Epidemiology, vol. 20, no. 4, pp. 302-307, 2010.

[18] C.-A. Liu, C.-L. Wang, H. Chuang, C.-Y. Ou, T.-Y. Hsu, and K. D. Yang, "Prenatal prediction of infant atopy by maternal but not paternal total IgE levels," Journal of Allergy and Clinical Immunology, vol. 112, no. 5, pp. 899-904, 2003.

[19] W. Eder, M. J. Ege, and E. Von Mutius, "The asthma epidemic," The New England Journal of Medicine, vol. 355, no. 21, pp. 22262235, 2006.

[20] E. Rönmark, A. Bjerg, M. Perzanowski, T. Platts-Mills, and B. Lundbäck, "Major increase in allergic sensitization in schoolchildren from 1996 to 2006 in Northern Sweden," Journal of Allergy and Clinical Immunology, vol. 124, no. 2, pp. 357-363, 2009.

[21] H. Bisgaard, A. Simpson, C. N. A. Palmer et al., "Geneenvironment interaction in the onset of eczema in infancy: 
filaggrin loss-of-function mutations enhanced by neonatal cat exposure," PLoS Medicine, vol. 5, no. 6, article el31, 2008.

[22] J. C. Burns, C. Shimizu, H. Shike et al., "Family-based association analysis implicates IL-4 in susceptibility to Kawasaki disease," Genes and Immunity, vol. 6, no. 5, pp. 438-444, 2005.

[23] C. L. Brosius, J. W. Newburger, J. C. Burns, P. Hojnowski-Diaz, S. Zierler, and D. Y. M. Leung, "Increased prevalence of atopic dermatitis in Kawasaki disease," Pediatric Infectious Disease Journal, vol. 7, no. 12, pp. 863-866, 1988.

[24] W. K. Liew, C. W. T. Lim, T. H. Tan et al., "The effect of Kawasaki disease on childhood allergies-a sibling control study," Pediatric Allergy and Immunology, vol. 22, no. 5, pp. 488-493, 2011.

[25] H.-C. Kuo, K. D. Yang, C.-D. Liang et al., "The relationship of eosinophilia to intravenous immunoglobulin treatment failure in Kawasaki disease," Pediatric Allergy and Immunology, vol. 18, no. 4, pp. 354-359, 2007.

[26] H. C. Kuo, C. L. Wang, C. D. Liang et al., "Association of lower eosinophil-related T helper 2 (Th2) cytokines with coronary artery lesions in Kawasaki disease," Pediatr Allergy Immunol, vol. 20, no. 3, pp. 266-272, 2009.

[27] H.-C. Kuo, C.-D. Liang, H.-R. Yu et al., "CTLA-4, position 49 A/G polymorphism associated with coronary artery lesions in Kawasaki disease," Journal of Clinical Immunology, vol. 31, no. 2, pp. 240-244, 2011.

[28] H.-C. Kuo, Y. Onouchi, Y.-W. Hsu et al., "Polymorphisms of transforming growth factor-B signaling pathway and Kawasaki disease in the Taiwanese population," Journal of Human Genetics, vol. 56, no. 12, pp. 840-845, 2011.

[29] G. Jones, S. Wu, N. Jang, D. Fulcher, P. Hogan, and G. Stewart, "Polymorphisms within the CTLA4 gene are associated with infant atopic dermatitis," British Journal of Dermatology, vol. 154, no. 3, pp. 467-471, 2006.

[30] S. Y. Choi, M. H. Sohn, B. C. Kwon, and K.-E. Kim, "CTLA-4 expression in T cells of patients with atopic dermatitis," Pediatric Allergy and Immunology, vol. 16, no. 5, pp. 422-427, 2005.

[31] M. Anthoni, G. Wang, C. Deng, H. J. Wolff, A. I. Lauerma, and H. T. Alenius, "Smad3 signal transducer regulates skin inflammation and specific IgE response in murine model of atopic dermatitis," Journal of Investigative Dermatology, vol. 127, no. 8, pp. 1923-1929, 2007.

[32] H.-C. Kuo, C.-L. Wang, C.-D. Liang et al., "Persistent monocytosis after intravenous immunoglobulin therapy correlated with the development of coronary artery lesions in patients with Kawasaki disease," Journal of Microbiology, Immunology and Infection, vol. 40, no. 5, pp. 395-400, 2007.

[33] J. Hirao, S. Hibi, T. Andoh, and T. Ichimura, "High levels of circulating interleukin-4 and intevleukin-10 in Kawasaki disease," International Archives of Allergy and Immunology, vol. 112, no. 2, pp. 152-156, 1997.

[34] J. Abe, R. Ebata, T. Jibiki, K. Yasukawa, H. Saito, and M. Terai, "Elevated granulocyte colony-stimulating factor levels predict treatment failure in patients with Kawasaki disease," Journal of Allergy and Clinical Immunology, vol. 122, no. 5, pp. 1008.e81013.e8, 2008.

[35] T. Matsubara, K. Katayama, T. Matsuoka, M. Fujiwara, M. Koga, and S. Furukawa, "Decreased interferon-gamma (IFN$\gamma$ )-producing T cells in patients with acute Kawasaki disease," Clinical and Experimental Immunology, vol. 116, no. 3, pp. 554557, 1999.
[36] A. Harnden, R. Mayon-White, R. Perera, D. Yeates, M. Goldacre, and D. Burgner, "Kawasaki disease in England: ethnicity, deprivation, and respiratory pathogens," Pediatric Infectious Disease Journal, vol. 28, no. 1, pp. 21-24, 2009.

[37] D. B. Peden, "The epidemiology and genetics of asthma risk associated with air pollution," Journal of Allergy and Clinical Immunology, vol. 115, no. 2, pp. 213-219, 2005.

[38] K.-S. Hsieh, T.-J. Lai, Y.-T. Hwang et al., "IL-10 promoter genetic polymorphisms and risk of Kawasaki disease in Taiwan," Disease Markers, vol. 30, no. 1, pp. 51-59, 2011.

[39] H.-C. Kuo and W.-C. Chang, "Genetic polymorphisms in Kawasaki disease," Acta Pharmacologica Sinica, vol. 32, no. 10, pp. 1193-1198, 2011.

[40] J.-H. Namkung, J.-E. Lee, E. Kim et al., "IL-5 and IL-5 receptor alpha polymorphisms are associated with atopic dermatitis in Koreans," Allergy, vol. 62, no. 8, pp. 934-942, 2007.

[41] S. A. Islam, D. S. Chang, R. A. Colvin et al., "Mouse CCL8, a CCR8 agonist, promotes atopic dermatitis by recruiting IL5+TH2 cells," Nature Immunology, vol. 12, no. 2, pp. 167-177, 2011.

[42] J. Corren, "Inhibition of interleukin-5 for the treatment of eosinophilic diseases," Discovery Medicine, vol. 13, no. 71, pp. 305-312, 2012.

[43] G. Lack, "Update on risk factors for food allergy," Journal of Allergy and Clinical Immunology, vol. 129, no. 5, pp. 1187-1197, 2012.

[44] B. S. Kim, M. C. Siracusa, S. A. Saenz et al., "TSLP elicits IL33-independent innate lymphoid cell responses to promote skin inflammation," Science Translational Medicine, vol. 5, no. 170, Article ID 170ra16, 2013.

[45] W.-C. Chang, C.-H. Lee, T. Hirota et al., "ORAI1 genetic polymorphisms associated with the susceptibility of atopic dermatitis in Japanese and Taiwanese populations," PLoS ONE, vol. 7, no. 1, Article ID e29387, 2012.

[46] H.-C. Kuo, Y.-J. Lin, S.-H. H. Juo et al., "Lack of association between ORAI1/CRACM1 Gene Polymorphisms and Kawasaki disease in the Taiwanese children," Journal of Clinical Immunology, vol. 31, no. 4, pp. 650-655, 2011.

[47] Y. Onouchi, T. Gunji, J. C. Burns et al., "ITPKC functional polymorphism associated with Kawasaki disease susceptibility and formation of coronary artery aneurysms," Nature Genetics, vol. 40, no. 1, pp. 35-42, 2008. 


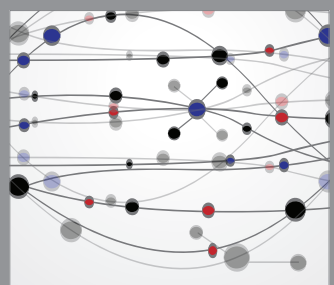

The Scientific World Journal
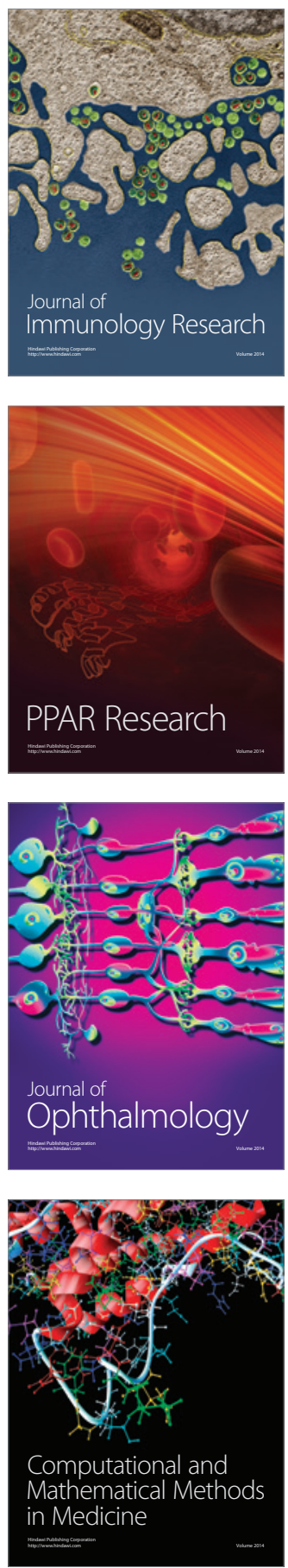

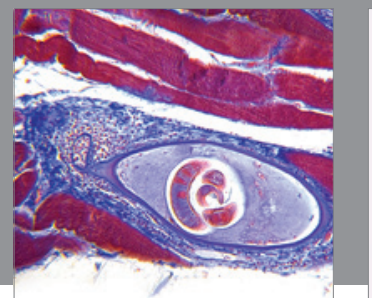

Gastroenterology

Research and Practice
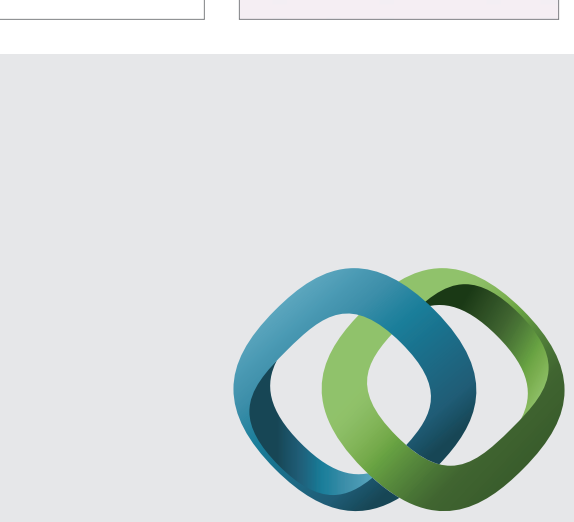

\section{Hindawi}

Submit your manuscripts at

http://www.hindawi.com
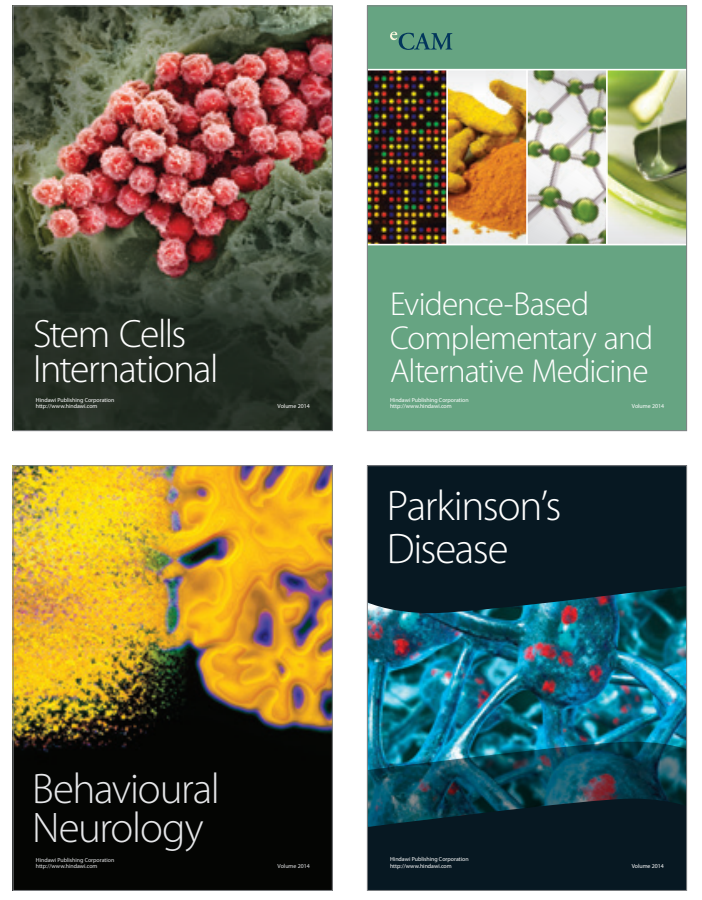
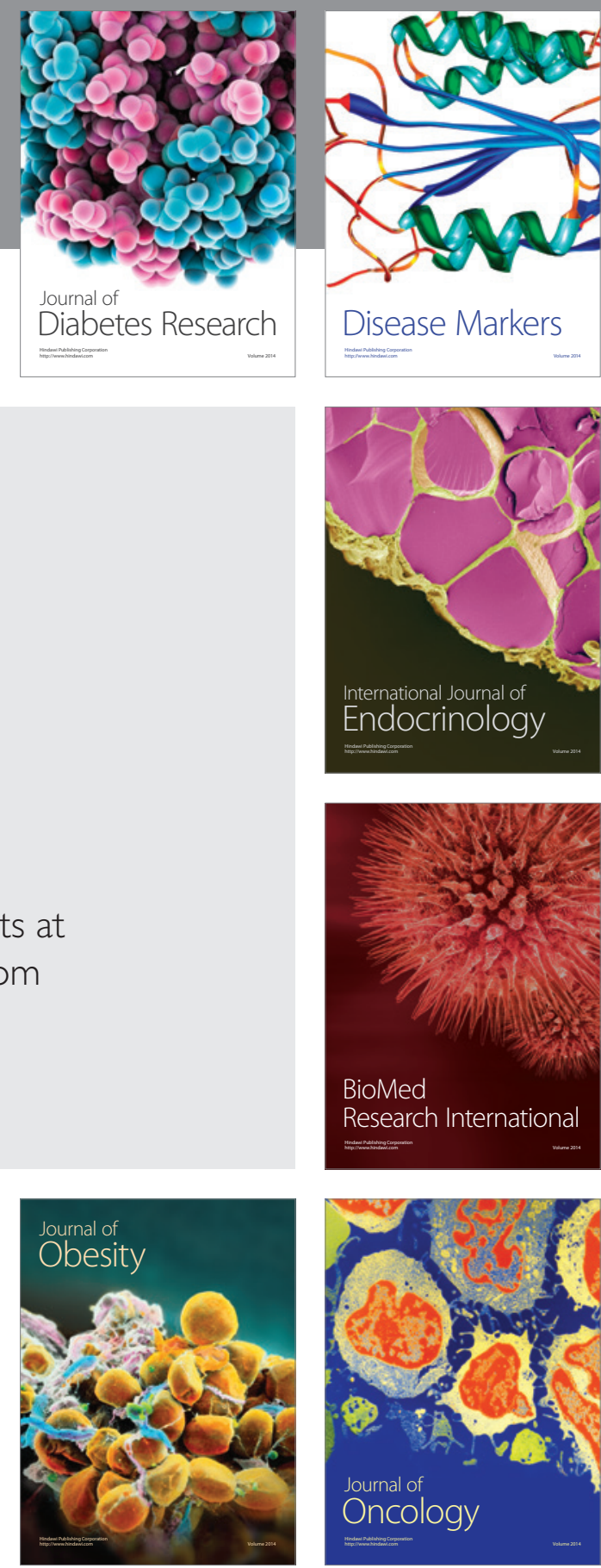

Disease Markers
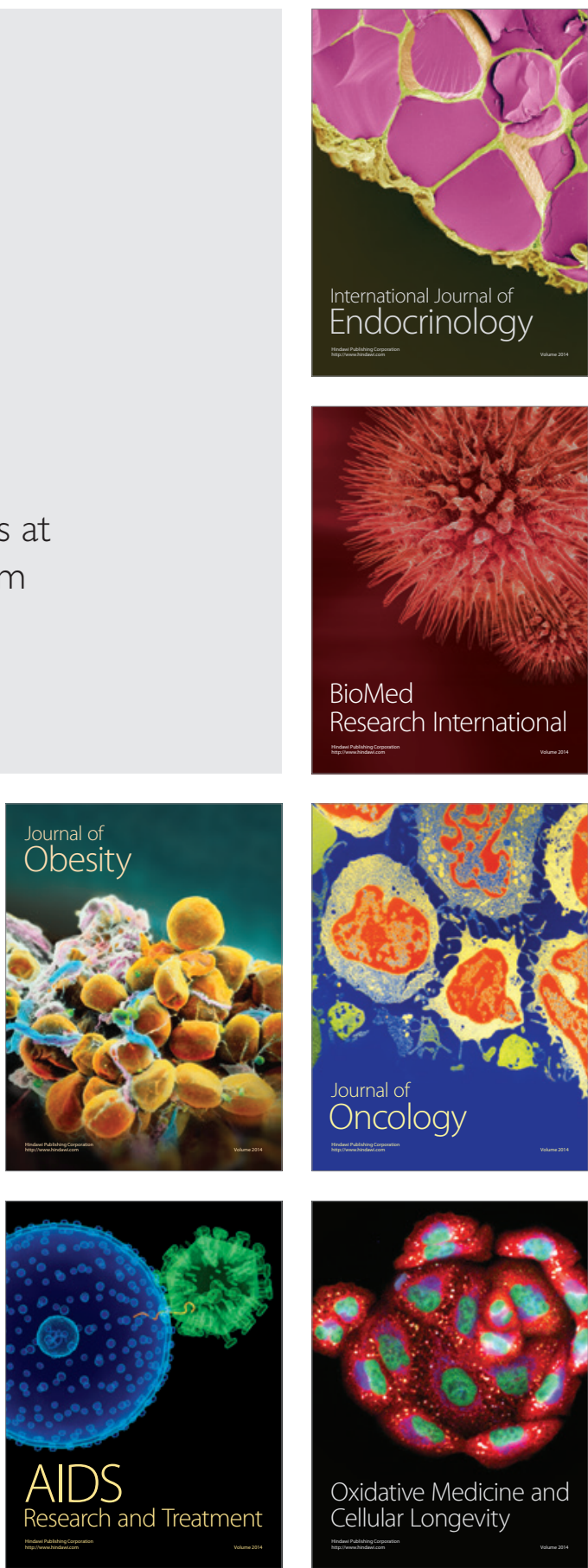